\title{
Alginate-Amphothericin B Nanocomplexes Covered By Nanocrystals From Bacterial Cellulose: Physico- Chemical Characterization And In Vitro Toxicity
}

Victória Soares Soeiro

Universidade de Sorocaba

Ricardo Silva-Carvalho

University of Minho

Daniela Martins

University of Minho

Pier Parpot

University of Minho

Denise Grotto

Universidade de Sorocaba

Marco Vinicius Chaud

Universidade de Sorocaba

Francisco Miguel Portela Gama

University of Minho

Angela Jozala ( $\sim$ angela.jozala@prof.uniso.br)

Universidade de Sorocaba

\section{Research Article}

Keywords: Bacterial cellulose, Nanocrystals, Amphotericin B. Alginate, Nanocomplexes

Posted Date: February 15th, 2021

DOl: https://doi.org/10.21203/rs.3.rs-105741/v2

License: (c) (1) This work is licensed under a Creative Commons Attribution 4.0 International License.

Read Full License 


\section{Abstract}

Amphotericin $B(A m B)$ is a drug with anti-leishmanial and anti-fungal ability, but it has low water solubility and permeability, limiting its therapeutic use. Therefore, the incorporation of AmB into nanostructured systems could be profit. Nanostructured systems without surfactants have pharmacotechnical advantages such as amplify the water solubility and decrease the toxicity. For this reason, the present study aimed to produce a nanocomplex of alginate (Alg) with AmB covered by NCC in order to decrease the toxicity of AmB. This straightforward process allowed to obtain water soluble particles Furthermore, the ionic adsorption of the NCC into the Alg-AmB nanocomplex surface was confirmed by an increase in the particle size and a small surface charge decrease. The amorphous inclusion of $A m B$ complex into the polysaccharide chain network in both formulations. AmB in the nanocomplexes was in supper-aggregated form and showed good biocompatibility, being significantly less cytotoxic in vitro against kidney cells and significantly less hemolytic compared to the free-drug. The results indicated the Alg-AmB nanocomplex can be considered a non-toxic alternative to improve the AmB therapeutic effect. Furthermore, NCC coating of the nanocomplexes brought additional protection to the system without compromised the advantages attributed to the developed formulation.

\section{Background}

Amphotericin $B(A m B)$ is a polyene antibiotic used either as a gold standard therapy for fungal infections, once it does not cause microbial resistance ${ }^{1}$, or as a second-line treatment for visceral leishmaniasis ${ }^{2}$. Despite being a drug widely used for more than half a century, AmB presents several limitations such as low water solubility and low permeability, in addition to high toxicity, especially nephrotoxicity. ${ }^{3}$.

Different formulations containing AmB are marketed, such as AmBisome ${ }^{\circledR}$ and Abelcet ${ }^{\circledR}$, which are based on lipids and awfully expensive formulations. Therefore, drug delivery systems have been used nowadays in order to overcome AmB limitations and improve its therapeutic efficacy. Encapsulating the drug in a nanocomplex system was an alternative found to solve the product problems. ${ }^{4}$.

Nanocomplexes are self-organized structures composed of a polymer (in many cases natural polysaccharides) and the drug. This system has advantages such as nano scale, high stability, good dispersion in water, low toxicity, with excellent cost-benefit and does not use solvents. In the literature, there are several works using different types of polymers, such as dextrin ${ }^{5}$, gum arabic ${ }^{6}$, albumin ${ }^{7}$ and alginate ${ }^{8}$. Alginate (Alg) is a polymer extracted from natural sources, widely used in the pharmaceutical area and in tissue engineering. This material has several advantages, such as immune system activation, nontoxicity, biocompatibility and biodegradability ${ }^{9-11}$. In our research, in order to reinforce and cover the system Alg-AmB, bacterial cellulose nanocrystals (NCC) were employed.

NCC can be used as a reinforcement material to drug delivery systems, protecting them and enabling the administration of the drug by different routes ${ }^{12,13}$. NCC are produced by the action of cellulase in contact with bacterial cellulose. This material has a renewable and sustainable origin, with good cost-benefit, 
biocompatibility and biodegradability, besides presenting a good mechanical resistance, large surface area, low toxicity $10,14,15$.

Since there is no delivery system to AmB using NCC, we aimed to develop nanocomplexes of Alg-AmB, evaluating the NCC as a cover system in order to protect the system, analysing physic-chemical characterization and the toxicity of $\mathrm{AmB}$, assess by in vitro assays.

\section{Results}

\section{Physico-chemical characterization of nanocomplexes}

Size, index of polydispersity (PDI) and zeta potential of the Alg-AmB nanocomplexes and the nanocomplexes covered by NCC (Alg-AmB + NCC) are shown in table I.

Table I - Analysis of the size, PDI and zeta potential of Alg-AmB Alg-Amb+NCC)

\begin{tabular}{lll} 
NCC & Alg-AmB & Alg-AmB + NCC \\
& & \\
\hline $41.67 \pm 5.55$ & $258.87 \pm 10.41$ & $466.3 \pm 17.57$ \\
& & $0.420 \pm 0.05$ \\
$0.127 \pm 0.005$ & & \\
& $0.523 \pm 0.073$ & \\
\hline $7.57 \pm 2,26 \mathrm{mV}$ & $-62.93 \pm 2.02$ & $-55.75 \pm 1.23$ \\
& & \\
\end{tabular}

Both nanocomplexes maintained their size on the nanometer scale. However, there was a significant increase in the size of the Alg-AmB + NCC nanocomplex, possibly due to coating by the NCC. On the other hand, when NCC was added NCCin nanocomplexes, the polydispersity index decreased.

Zeta potential of the nanocomplexes increased in the sample containing NCC. The increase was indicated the possibility of nanocomplexes coating. Although the NCC have a positive characteristic, the value is very low; so the coating value has not changed significantly.

Figure 1 shows Differential Scanning Calorimetry (DSC) data for AmB, Alg, NCC, Alginate + AmB (physical mixture), Alg-AmB and Alg- AmB + NCC.

\section{Note}

A - DSC analysis of AmB, Alg, NCC, alginate + AmB (mixture), Alg-AmB and Alg-AmB + NCC. B - FTIR of pure alginate, Alg and Alg-AmB. D - UV-Vis spectrometry of the AmB, Alg-AmB and Alg-AmB + NCC.

In Amphotericin B sample, we have found a peak in $167^{\circ} \mathrm{C}$ degrees. For NCC sample, the peak was at 151

${ }^{\circ} \mathrm{C}$. The physical formation of AmB-alginate mixture showed peaks at $144^{\circ} \mathrm{C}$, referring to alginate, and at 
$161^{\circ} \mathrm{C}$ referring to $\mathrm{AmB}$. On the other hand, when Alg-AmB nanocomplexes were formed, DSC showed only one peak, at $141^{\circ} \mathrm{C}$, for alginate.

The DSC value for Alg-AmB + NCC sample showed a similar peak to Alg-AmB, but with a slightly lower temperature, at $123^{\circ} \mathrm{C}$. This indicates when NCC was added in the formulation, there was a decrease in the temperature.

Figure 1.B shows the FTIR data of the samples of pure alginate, Alg and Alg-AmB. The wavelength band $3424 \mathrm{~cm}^{-1}$ presented in all samples refers to the $\mathrm{O}-\mathrm{H}$ stretching vibrations of the alginate. The weak band at $2930 \mathrm{~cm}^{-1}$ represents the $\mathrm{C}-\mathrm{H}$ stretching vibrations; the bands 1630 and $1416 \mathrm{~cm}^{-1}$ are asymmetrical and symmetrical stretching vibrations of the $\mathrm{C} \equiv \mathrm{O}$ and the COO- group. Furthermore, the band around $1024 \mathrm{~cm}^{-1}$ refers to the vibrations of the ring elongation $\mathrm{C}-\mathrm{O}$ and $\mathrm{C}-\mathrm{O}$, with deformations of $\mathrm{C}-\mathrm{C}-\mathrm{H}$ and $\mathrm{C}$ $\mathrm{O}-\mathrm{H}^{8,16}$. In the Alg-AmB sample, the AmB wavelength bands were compared to data from the literature, showing the bands: $\mathrm{OH}$ stretching vibration $\left(3434 \mathrm{~cm}^{-1}\right), \mathrm{CH}$ flexion vibrations and $\mathrm{CH}_{3}$ oscillation (1024 $\left.\mathrm{cm}^{-1}\right)^{17}$. The hypothesis is the other bands are overlapped by the alginate bands.

The Fig. 1.D shows the UV-vis spectra of AmB, Alg-AmB nanocomplex and Alg-AmB + NCC.

In this study, AmB was firstly dissolved in $0.1 \mathrm{M}$ borate buffer $\mathrm{pH} 11$ and then diluted in water, in a monomeric state. The ratio $\mathrm{Abs}_{349} / \mathrm{Abs}_{410}$ was 0.36 . Considering the Alg-AmB nanocomplex, the1st peak $(327 \mathrm{~nm})$ and the ratio between the peaks I and IV $\left(\mathrm{Abs}_{327} / \mathrm{Abs}_{410}\right)$ was 4.38 , showing AmB is in a supper-aggregated state in the formulation, which, as referred above, is associated to a low toxicity. Despite promoting a decrease in the absorbance intensity, NCC addition to the nanocomplex surface did not affect the supper-aggregated state of $\mathrm{AmB}$ since a ratio of $4.45\left(\mathrm{Abs}_{327} / \mathrm{Abs}_{409}\right)$ was found.

\section{In vitro toxicity}

The hemolysis test was performed for AmB, Alg, Alg-AmB, Alg-AmB + NCC and AmBisome, in concentrations of $1,2,4,8,16$ and $32 \mu \mathrm{M}$ (Fig. 2.A).

\section{Note}

$A-A m B(*)$ and Alg-AmB (**) were compared to the other groups by the Duncan test $(p<0,05)$. B - AmB $\left.{ }^{*}\right)$ was compared to the other groups by the Duncan test $(p<0.05)$.

The standard sample of Alg showed irrelevant values of hemolysis (lower than $2 \%$ ), in all concentration. AmB induced hemolysis percentage between $1.97 \pm 1.66 \%$ and $81.18 \pm 12.50 \%$. When alginate was added to the formulation (Alg-AmB), there was a decrease in toxicity of $90 \%$ compared to standard AmB, with hemolysis values from $-0.08 \%$ to $30.25 \pm 0.50 \%$. When Alg-Amb was coated with NCC, the values were similar $(-0.3 \pm 0.19 \%$ and $44.7 \pm 0.50 \%)$. Even at high concentrations, nanocomplexes reduced the rate of hemolysis in comparison to standard AmB. 
Regarding hemolytic porcetange of AmBisome $\AA$, data ranged from $0.34 \pm 0.33 \%$ to $2.13 \pm 1 \%$. Although AmBisome's hemolysis values are lower than those found in the Alg-AmB formulation, Alg-AmB nanocomplexes had excellent results compared with free AmB. Hemolysis diminish in the sample due to the incorporation of AmB in Alg nanocomplexes shows the nanocomplexes are safe.

In Fig. 2.B is presented the cytotoxicity data for AmB, Alg, Alg-AmB, Alg-AmB + NCC and AmBisome $\AA$. Alg showed cell viability between $93.01 \pm 3.83 \%$ and $108.66 \pm 7.04 \%$. AmB data indicated concentrationdependent toxicity. On the other hand, the Alg-AmB nanocomplexes demonstrated significant less toxicity than free AmB. Cell viability was high, ranging between $97.16 \pm 5.61 \%$ and $109.86 \pm 7.06 \%$.

For the Alg-AmB + NCC nanocomplex, the cell viability values were similar to Alg-AmB NCC, ranging from $98.55 \pm 5.06 \%$ to $106.40 \pm 5.35 \%$, demonstrating safety use of coating with NCC. Cell viability for AmBisome ${ }^{\circledR}$ was around $101.40 \pm 6.23 \%$ to $111.29 \pm 6.92 \%$. When comparing the results of the commercial sample, AmBisome ${ }^{\circledR}$, with Alg-AmB and Alg-AmB + NCC nanocomplexes, there was no significant difference among them, indicating both nanocomplexes, with or without NCC, are as safe as the commercial one.

\section{Discussion}

In this work, alginate-amphotericin nanocomplexes (Alg-Amb) were developed aiming to improve the AmB water solubility with low toxicity. It was also evaluated the influence of NCC as a cover system to protect the system. Therefore, to achieve this Aim, it was realized physico-chemical characterization and in vitro toxicity.

Taheri and Mohammadi (2015) ${ }^{18}$ utilized cellulose nanocrystals to cover a hydroquinone system and they demonstrated an increase in particle size. The way to obtain smaller particles is important, because expanding the routes of administration. Systems with polymers nanoparticles of size around $200 \mathrm{~nm}$ are absorbed by the spleen, lung and liver ${ }^{4}$.

The nanocomplexes method is capable of producing smaller particles than ionotropic gelification method. Senna et al. (2018) ${ }^{19}$ produced particles by ionotropic gelation and observed the production of particles of $1.2 \pm 0.34 \mathrm{~mm}$. Compared to our method; these particles were about 3 times larger than the nanocomplex coated by NCC. One study with alginate particles containing miltefosine obtained by ionotropic gelation and emulsification method showed a polydispersity index of $0.43 \pm 0.14{ }^{20}$, similar to the data found in our study.

Comparatively in another study, the zeta potential of alginate particles obtained by ionotropic gelation showed negative values in the range of $-15.7 \pm 1.7$ and $-9.8 \pm 1.2 \mathrm{mV}$. However, when adding chitosan in the formulation, there was a change in zeta potential due to the coating 21 .

$A m B$ is characterized by two endothermic peaks in the crystalline form ${ }^{22}$. In our study, AmB standard showed peaks at $167^{\circ} \mathrm{C}$ (melting point) and $198^{\circ} \mathrm{C}$, similar to the temperatures found by those authors. 
Alginate showed an endothermic peak at $149^{\circ} \mathrm{C}$, which demonstrates the degradation of the biopolymer 23,24. Vasconcelos et al. $2017{ }^{25}$. reported peaks to NCC obtained by acid hydrolysis ranging from 140 to $195^{\circ} \mathrm{C} 25$, similar to our finding (peak at $151^{\circ} \mathrm{C}$ ). Regarding Alg-AmB nanocomplexes, we have described only one peak. The absence of AmB peak indicates there was a change from crystalline to amorphous state, demonstrating the formation and improvement of alginate and AmB nanocomplexes ${ }^{26}$.

In a study with alginate associated to the polymer poly [N-(2-hydroxypropyl) methacrylamide] and the drug camptothecin, the characteristic peak of camptothecin was absent due to the drug being compatible with the polymers and its high dispersion in the formulation ${ }^{27}$. About alginate sample, similar results were found by Silva-Carvalho et al. (2020), indicating the importance of using safe polysaccharides for the formation of the nanocomplex. Another examples can be observed in a study whose objective was to use NCC as a reinforcement for a film containing polylactic acid, concluding there was an increase in crystallinity ${ }^{28}$. George et al., (2011 reported the utilization of NCC associated with polymers and showed there was an increase in the parameters of glass transition, melting temperature, enthalpy and crystalline behaviour.

The degree of AmB aggregation can be determined by the ratio from the first to the fourth peak in the UV-Vis spectra, being a value of $<1$ related to the monomeric form and a value of $>2$ related to a supperaggregated form ${ }^{4,6,8,29}$. In solution, AmB has three different states that affect its activity and pharmacokinetical characteristics, such as monomers, in which AmB is water soluble normally associates with ergosterol in fungal and protozoan cell membranes; oligomers, a state in which small water aggregates are toxic towards host cells and present very low solubility; and poly-aggregates, that also referred as supper-aggregates present low in vitro and in vivo toxicity ${ }^{6,8}$.

Fungizone ${ }^{\circledR}$ and $A m B i s o m e \AA$ are drugs with low toxicity found in the pharmaceutical market. Due to the super-aggregated state of AmB, specified by ratio values of 2.9 and 4.8 (Alg-AmB and Alg-AmB+NCC, respectively), our nanocomplexes have similar data in relation to commercial formulations ${ }^{7,30}$.

The hemolysis test is important in studies with nanocomplexes or nanoparticles containing AmB once there is evidence of hemolytic anemia caused by the use of free $A m B{ }^{31,32}$. AmB has a high hemotoxicity even at low concentrations, due to the aggregated conformation ${ }^{31}$. Conjugates and ionic cross-linked polymeric nanoparticles of AmB with alginate show less hemotoxicity and greater hemocompatibility than free AmB, possibly by the protective effect from polysaccharide and by the change in conformation of the super-aggregated state ${ }^{4,8}$.

The importance of cytotoxicity test with this cell strain is due to the nephrotoxicity associated with free $A m B{ }^{7}$. Therefore, it is important to understand the behaviour of nanocomplexes in this type of cells. The non-toxicity and the capacity for cell proliferation observed to alginate experimentation have already been described ${ }^{4}$. In a study, AmB solution at $15.6 \mu \mathrm{g} / \mathrm{mL}$ was able to induce death in renal cells ${ }^{17}$. On the other hand, Ravichandran and Jayakrishnan, 2018 tested AmB and alginate conjugates to verify cytotoxicity and observed a decrease in toxicity compared to free AmB. 
Although there are studies reporting reduction in hemo and cytotoxicity of amphotericin, our work proposes a simplified methodology, with a cost-benefit and achieves the same objectives by complex and high cost methodologies. Therefore, in addition to our material being as safe as the standard medicine (AmBisome ${ }^{\circledR}$ ). As,Silva-Carvalho, et al, 2020 the findings, our work, overall, demonstrates the suitability of alginate as an AmB carrier, covered or not by NCC.

\section{Conclusion}

In our study, the development of an alginate nanocomplexes with AmB decreased the hemolytic and cytotoxic effect of the drug. In addition, coating the nanocomplexes with the NCC, produced by enzymatic way, brought additional protection to the system without compromised the advantages attributed to the developed formulation, which can be considered a cost benefit, non-toxic and safe as the commercial one. These findings have an important relevance once when developing a nanocomplex, the great advantage is to direct the formulation to the drug's action sites nd with the extra cover of NCC can be applied in other administration route.

\section{Methodology}

\section{Reagents}

Amphotericin B (AmB, molecular weight of $924.08 \mathrm{~g} / \mathrm{mol})$ powder from Streptomyces sp., resazurin Sodium salt, sodium alginate, triton X-100, sodium tetraborate decahydrate were purchased from SigmaAldrich (Missouri, USA). Dulbecco's Modified Eagle Medium (DMEM), fetal bovine serum (FBS) and penicillin-streptomycin were obtained from Merck Millipore (Massachusetts, USA). Roswell Park Memorial Institute (RPMI) 1640. Glutamax supplemented medium and L-glutamine (GlutaMAX ${ }^{\mathrm{TM}}-\mathrm{I}$ ) was purchased from Gibco (Massachusetts, USA). Dimethylsulfoxide (DMSO ATCC ${ }^{\circledR} 4-X^{T M}$ ) solution for cell culture was acquired from American Type Culture Collection (ATCC, Virginia, USA). Dialysis tubing with a molecular weight cut-off of 1000 Da was obtained from Orange Scientific (Braine-l'Alleud, Belgium). AmBisome ${ }^{\circledR}$ was kindly provided by Gilead Sciences.

\section{Obtaining bacterial cellulose nanocrystals (NCC)}

NCC production was carried out following the methodology developed by our research group (In presssupplementary material). The bacterial cellulose was obtained from G. xylinus, according to Jozala et al. (2014) ${ }^{33}$.The process uses a mechanical treatment of bacterial cellulose with ultra-turrax and highpressure homogenizer and a enzymatic treatment with cellulase for 72 hours. After, the NCC were separated with centrifugation and filtration.

\section{Preparation of alginate-amphotericin B (Alg-AmB) nanocomplexes}

The Alg-AmB nanocomplexes were prepared according to Silva-Carvalho et al. (2020) ${ }^{5}$. In the formulation, borate buffer ( $\mathrm{pH}$ 11.0), sodium alginate and amphotericin B were used, resulting in a final 
concentration of $2.5 \mathrm{mg} / \mathrm{mL}$ of $\mathrm{AmB}$ in the solution. The nanocomplexes formulation were described in table II.

Table II - Formulation of alginate (Alg) and alginate-amphotericin B (Alg-AmB).

\begin{tabular}{l|lll}
\hline Formulations & Borate buffer $\mathbf{p H} 11(\mathbf{m L})$ & Sodium alginate $(\mathbf{m g})$ & Amphotericin $\boldsymbol{B}(\mathbf{m g})$ \\
\hline$A l g$ & 9.6 & 120 & - \\
$A l g-A m B$ & 9.6 & 96 & 24 \\
\hline
\end{tabular}

The samples were kept under agitation at $4{ }^{\circ} \mathrm{C}$ for 48 hours without interference from light. In the next step, the dialysis process was run on a membrane from 12000 to $14000 \mathrm{KDa}$. The membrane with the sample was immersed in $5 \mathrm{~L}$ of distilled water under agitation at $4{ }^{\circ} \mathrm{C}$, without light interference, for 30 hours. During the process, the water was changed three times to remove salts, until reaching $\mathrm{pH}$ value in the range of 5.5-5.7. Then, the samples were collected, frozen at $-80^{\circ} \mathrm{C}$ for 24 hours, and lyophilized. The final yield of the process was $73.72 \%$.

\section{Nanocomplex coating}

Alg and Alg-AmB nanocomplexes were covered with NCC. For that, the lyophilized nanocomplexes were dispersed in water in the proportion of $1 \mathrm{mg} / \mathrm{mL}$. After dispersion, $1 \mathrm{ml}$ of the nanocomplexes was added into $1 \mathrm{ml}$ of $0.01 \% \mathrm{NCC}$ suspension. This sample was kept on rotatory shaker $(20 \mathrm{rpm})$ at $25^{\circ} \mathrm{C}$ for 24 hours.

\section{Physico-chemical characterization}

For the physico-chemical characterization the following parameters were used: size, index of polydispersity, zeta potential, differential scanning calorimetry, infrared spectrometry by Fourier transform (FTIR) and UV-Vis spectrometry.

For the evaluation of the size, index of polydispersity and surface potential of the particles (zeta potential), the Zetasizer equipment (ZEN3600) was used, at an angle of $173^{\circ}$, at $25^{\circ} \mathrm{C}$ for dynamic light scattering (DLS), Index of Polydispersity and zeta potential. The samples Alg-AmB and Alg-AmB + NCC were analysed in six times.

To characterize the stability of the nanocomplexes, Differential Scanning Calorimetry (DSC) was used. The characterization was performed by the DSC 6000 equipment (PERKIN ELMER I STEC INSTRUMENTS) in a nitrogen atmosphere with a flow of $20 \mathrm{~mL} / \mathrm{min}$, in the heating range of $25-250{ }^{\circ} \mathrm{C}$ with a heating rate of $10{ }^{\circ} \mathrm{C} / \mathrm{min}$. The test was re-performed with the samples AmB, Alg nanocomplexes, 
$\mathrm{NCC}$, alginate $+\mathrm{AmB}$ (physical mixture without nanocomplexes process), Alg-AmB nanocomplex and Alg$\mathrm{AmB}+\mathrm{NCC}$.

The characterization of the chemical groups was carried out by spectrometry in FTIR. The samples were analysed by the technique of $\mathrm{KBr}$ tablets, with $2 \mathrm{mg}$ of sample and $200 \mathrm{mg}$ of $\mathrm{KBr}$. The infrared spectra were obtained in the range of $4000-400 \mathrm{~cm}^{-1}$ in the Bruker Alpha II equipment, with a resolution of $4 \mathrm{~cm}^{-1}$ and 12 scans. The samples analysed were: pure alginate, Alg nanocomplexes and Alg-AmB.

In addition, the samples were analysed by UV-Vis spectroscopy in the 300-450 nm range using a UVVisible spectrophotometer (JASCO V-560) with $5 \mathrm{~nm}$ resolution and scanning speed of $400 \mathrm{~nm} / \mathrm{min}$. The nanocomplexes Alg and Alg-AmB were dispersed in distilled water (1 $\mathrm{mg}$ of sample/ $\mathrm{mL}$ ). The material was diluted again to $10 \mu \mathrm{M}$ in distilled water in order to avoid saturation of the test. AmB control was performed using $1082 \mu \mathrm{M}$ diluted in borate buffer $(0.1 \mathrm{M}, \mathrm{pH} 11)$. The solution was also diluted to $10 \mu \mathrm{M}$ with distilled water. To perform the measurement, $200 \mu \mathrm{L}$ of the solutions were analyzed in a quartz cuvette. The absorbance ratio of peak I (315-350) to peak IV (408-410) was used to monitor the aggregation status of $A m B$ and the results were evaluated for the quantification of $A m B$ in the sample.

\section{In vitro toxicity}

For the in vitro toxicity tests, a commercial product (AmBisome ${ }^{\circledR}$ ) was taken as a control. This drug is for injectable use and it has a liposomal formulation containing AmB. AmBisome ${ }^{\circledR}$ prescription is mentioned in several articles due to its low toxicity ${ }^{6,7,34}$. Hemolysis and cytotoxicity were the testes chosen to perform the safety of the new nanocomplexes.

\section{Hemolysis test}

Hemolysis test assesses the percentage of erythrocyte rupture. Both the protocol and the animal's blood were derived from Silva-Carvalho et al. (2020) ${ }^{5}$. We used ordinary blood samples (dog) from a blood bank. The whole blood was centrifuged for $10 \mathrm{~min}\left(4^{\circ} \mathrm{C}, 1200 \mathrm{~g}\right)$ and the supernatant was discarded. Red blood cells were resuspended in phosphate buffer solution $(\mathrm{pH} \mathrm{7.4)}$.

Resuspended red blood cells were counted in a Neubauer chamber. In a 48-well plate, $450 \mu \mathrm{L}$ of red blood cells at a concentration of $1 \times 10^{8}$ cells / $\mathrm{mL}$ were placed in contact with $50 \mu \mathrm{L}$ of the samples, at concentrations of 1, 2, 4, 8, 16 and $32 \mu \mathrm{M} \mathrm{AmB}$, Alg, Alg -AmB, Alg-AmB + NCC and AmBisome ${ }^{\circledR}$. The plate was incubated with shaking for 30 minutes $\left(37^{\circ} \mathrm{C}, 120 \mathrm{rpm}\right)$.

After the period, the solutions were collected and centrifuged for $10 \mathrm{~min}\left(4^{\circ} \mathrm{C}, 1200 \mathrm{G}\right)$. The supernatants were collected and analyzed by UV-Vis spectrophotometry, with absorbance at $540 \mathrm{~nm}$ (referring to hemoglobin). Complete lysis (100\%) was admitted by hemoglobin released with $1 \%$ triton X-100 (positive control).

Cytotoxicity 
Human Embryonic Kidney (HEK) cell line was selected to perfume citotoxity due to the toxicity of AmB in renal cells. The HEK 293T monolayer $\left(1 \times 10^{4}\right.$ cell/well) was incubated for $24 \mathrm{~h}\left(37^{\circ} \mathrm{C}\right.$ in a $5 \% \mathrm{CO}_{2}$ atmosphere) with AmB, Alg, Alg-AmB, Alg-AmB + NCC and AmBisome ${ }^{\circledR}$ at concentrations of $0.78,1,17$, $1.76,2.63,3.95,5.93,8.89,13.33,20,30,45$ and $67.5 \mu \mathrm{M}$. After the incubation period, $10 \%(\mathrm{v} / \mathrm{v})$ of a 2.5 $\mathrm{mM}$ resazurin solution was added to each well and the plates were incubated again under the same conditions as above for 4 hours.

Fluorescence was measured ( $\lambda 560$ / $\lambda$ 590) in a SpectraMAX GeminiXS microplate reader (Molecular Devices LLC, California, USA). The results were expressed as mean percentage \pm SD of viable cells in relation to the positive control (condition considered to be $100 \%$ viable cells).

\section{Statistical analysis}

Data from toxicity tests were expressed as mean \pm standard deviation. Analysis of Variance (ANOVA) followed by the Duncan test were used to verify differences among treatment protocols, and $p$ values $<0.05$ were considered significant. Results were analyzed using Statistica ${ }^{\circledR}$ v. 8.0 (Dell, Round Rock, TX, USA) and GraphPad Prism® v. 6.0 (San Diego, CA, USA).

\section{Declarations}

\section{Acknowledgements}

The authors acknowledge financial support from Coordination for Higher Level Graduate Improvements (CAPES/Brazil, finance code 001), National Council for Scientific and Technological Development (CNPq/Brazil Process \#428751/2016-4), and the State of São Paulo Research Foundation (FAPESP/Brazil, processes \#2019/22626-5 and \#2018/10508-5).

\section{Author contributions}

VSS: Investigation, Methodology, Formal analysis, Writing - original draft; RSC: Investigation, Methodology, Formal analysis, Writing - review \& editing; DM: Methodology develop; PP: Methodology develop; DG: Validation, Writing - review \& editing; MVC: Writing - review \& editing; FMG: Conceptualization, Writing - review \& editing, Supervision; AFJ: Conceptualization, Writing - review \& editing, Supervision.

\section{Additional Information}

Competing Interests Statement: the authors declare that they have no competing interests

\section{References}

1. Gray, K. C. et al. Amphotericin primarily kills yeast by simply binding ergosterol. Proc. Natl. Acad. Sci. U. S. A. 109, 2234-2239 (2012). 
2. TORRADO, J. J., ESPADA, R., BALLESTEROS, M. P. \& TORRADO-SANTIAGO, S. Amphotericin B Formulations and Drug Targeting. J. Pharm. Sci. 97, 2405-2425 (2008).

3. Richter, A. R., Feitosa, J. P. A., Paula, H. C. B., Goycoolea, F. M. \& de Paula, R. C. M. Pickering emulsion stabilized by cashew gum- poly-L-lactide copolymer nanoparticles: Synthesis, characterization and amphotericin B encapsulation. Colloids Surfaces B Biointerfaces 164, 201-209 (2018).

4. Gupta, P. K. et al. Self Assembled lonically Sodium Alginate Cross-Linked Amphotericin B Encapsulated Glycol Chitosan Stearate Nanoparticles: Applicability in Better Chemotherapy and NonToxic Delivery in Visceral Leishmaniasis. Pharm. Res. 32, 1727-1740 (2015).

5. Silva-Carvalho, R. et al. Development of dextrin-amphotericin B formulations for the treatment of Leishmaniasis. Int. J. Biol. Macromol. 153, 276-288 (2020).

6. Nishi, K. K. et al. Amphotericin B-gum arabic conjugates: Synthesis, toxicity, bioavailability, and activities against Leishmania and fungi. Pharm. Res. 24, 971-980 (2007).

7. Gurudevan, S., Francis, A. P. \& Jayakrishnan, A. Amphotericin B-albumin conjugates: Synthesis, toxicity and anti-fungal activity. European Journal of Pharmaceutical Sciences vol. 115 (Elsevier B.V, 2018).

8. Ravichandran, V. \& Jayakrishnan, A. Synthesis and evaluation of anti-fungal activities of sodium alginate-amphotericin B conjugates. Int. J. Biol. Macromol. 108, 1101-1109 (2018).

9. Ng, W. Y., Migotto, A., Ferreira, T. S. \& Lopes, L. B. Monoolein-alginate beads as a platform to promote adenosine cutaneous localization and wound healing. Int. J. Biol. Macromol. 102, 1104-1111 (2017).

10. Yan, H. et al. A novel and homogeneous scaffold material: preparation and evaluation of alginate/bacterial cellulose nanocrystals/collagen composite hydrogel for tissue engineering. Polym. Bull. 75, 985-1000 (2018).

11. Thi, N. et al. Fabrication of alginate microspheres for drug delivery: A review. Int. J. Biol. Macromol. 153, 1035-1046 (2020).

12. Lee, J. \& Cheng, Y. Critical freezing rate in freeze drying nanocrystal dispersions. J. Control. Release 111, 185-92 (2006).

13. Yan, H. et al. Biointerfaces Entrapment of bacterial cellulose nanocrystals stabilized Pickering emulsions droplets in alginate beads for hydrophobic drug delivery. Colloids Surfaces B Biointerfaces 177, 112-120 (2019).

14. George, J., Ramana, K. V., Bawa, A. S. \& Siddaramaiah. Bacterial cellulose nanocrystals exhibiting high thermal stability and their polymer nanocomposites. Int. J. Biol. Macromol. 48, 50-57 (2011).

15. George, J. \& Sabapathi, S. N. Cellulose nanocrystals: Synthesis, functional properties, and applications. Nanotechnol. Sci. Appl. 8, 45-54 (2015).

16. Larosa, C. et al. International Journal of Biological Macromolecules Characterisation of bare and tannase-loaded calcium alginate beads by microscopic, thermogravimetric , FTIR and XRD analyses. Int. J. Biol. Macromol. 115, 900-906 (2018). 
17. Francis, A. P., Gurudevan, S. \& Jayakrishnan, A. Synthetic polymannose as a drug carrier: synthesis, toxicity and anti-fungal activity of polymannose-amphotericin B conjugates. Journal of Biomaterials Science, Polymer Edition vol. 29 (Taylor \& Francis, 2018).

18. Taheri, A. \& Mohammadi, M. The use of cellulose nanocrystals for potential application in topical delivery of hydroquinone. Chem. Biol. Drug Des. 86, 882-886 (2015).

19. Senna, J. P. et al. Dual alginate-lipid nanocarriers as oral delivery systems for amphotericin B. Eur. J. Pharm. Sci. 32, 187-194 (2018).

20. Spadari, C. de C., de Bastiani, F. W. M. da S., Lopes, L. B. \& Ishida, K. Alginate nanoparticles as nontoxic delivery system for miltefosine in the treatment of candidiasis and cryptococcosis. Int. J. Nanomedicine 14, 5187-5199 (2019).

21. Adebisi, A. O. \& Conway, B. R. Preparation and characterisation of gastroretentive alginate beads for. J. Microencapsul. 31, 58-67 (2013).

22. Zu, Y. et al. Preparation and characterization of amorphous amphotericin B nanoparticles for oral administration through liquid antisolvent precipitation. Eur. J. Pharm. Sci. 53, 109-117 (2014).

23. Sarmento, B., Ferreira, D., Veiga, F. \& Ribeiro, A. Characterization of insulin-loaded alginate nanoparticles produced by ionotropic pre-gelation through DSC and FTIR studies. Carbohydr. Polym. 66, 1-7 (2006).

24. Patel, M. A., AbouGhaly, M. H. H., Schryer-Praga, J. V. \& Chadwick, K. The effect of ionotropic gelation residence time on alginate cross-linking and properties. Carbohydr. Polym. 155, 362-371 (2017).

25. Vasconcelos, N. F. et al. Bacterial cellulose nanocrystals produced under different hydrolysis conditions: Properties and morphological features. Carbohydr. Polym. 155, 425-431 (2017).

26. Al-quadeib, B. T., Radwan, M. A., Horrocks, B. \& Wright, M. C. Stealth Amphotericin B nanoparticles for oral drug delivery: In vitro optimization. Saudi Pharm. J. 23, 290-302 (2015).

27. Mohanraj, S. \& Rajiv, S. Preparation and characterization of camptothecin-loaded alginate/poly[N-(2hydroxypropyl) methacrylamide] hydrogel beads for anticancer treatment. Int. J. Polym. Mater. Polym. Biomater. 66, 781-790 (2017).

28. Wardhono, E. Y., Kanani, N. \& Rahmayetty, A. \&. Development of polylactic acid ( PLA ) biocomposite films reinforced with bacterial cellulose nanocrystals (BNCC) without any surface modification. J. Dispers. Sci. Technol. 0, 1-8 (2019).

29. Barwicz, J., Christian, S. \& Gruda, I. Effects of the aggregation state of amphotericin B on its toxicity to mice. Antimicrob. Agents Chemother. 36, 2310-2315 (1992).

30. Mullen, A. B. \& Carter, K. C. Comparison of the Efficacies of Various Formulations of Amphotericin B against Murine Visceral Leishmaniasis. Antimicrob. Agents Chemother. 41, 2089-2092 (1997).

31. Vásquez Marcano, R. G. del J., Tominaga, T. T., Khalil, N. M., Pedroso, L. S. \& Mainardes, R. M. Chitosan functionalized poly ( $\varepsilon$-caprolactone) nanoparticles for amphotericin B delivery. Carbohydr. Polym. 202, 345-354 (2018). 
32. Jain, J. P. \& Kumar, N. Development of amphotericin B loaded polymersomes based on (PEG)3-PLA co-polymers: Factors affecting size and in vitro evaluation. Eur. J. Pharm. Sci. 40, 456-465 (2010).

33. Jozala, A. F. et al. Bacterial cellulose production by Gluconacetobacter xylinus by employing alternative culture media. Appl. Microbiol. Biotechnol. 99, 1181-1190 (2014).

34. Serrano, D. R. et al. Hemolytic and pharmacokinetic studies of liposomal and particulate amphotericin B formulations. Int. J. Pharm. 447, 38-46 (2013).

\section{Figures}



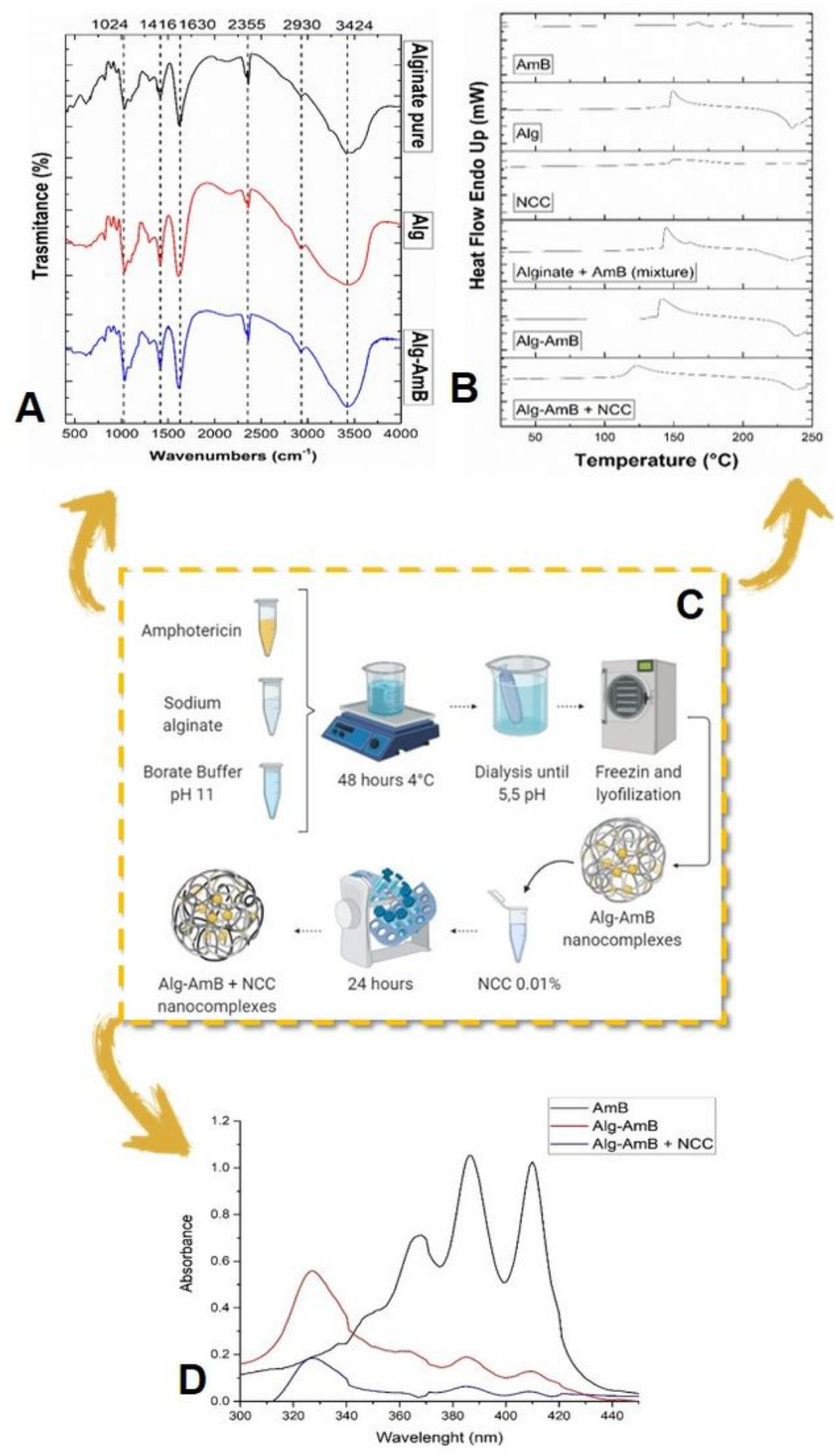

\section{Figure 1}

A - Differential Scanning Calorimetry (DSC). B - Infrared spectrum by Fourier transform (FTIR). C Design Production of Alg-AmB and Alg-AmB + NCC. D - UV-Vis spectrometry. Note: A - DSC analysis of AmB, Alg, NCC, alginate + AmB (mixture), Alg-AmB and Alg-AmB + NCC. B - FTIR of pure alginate, Alg and Alg-AmB. D - UV-Vis spectrometry of the AmB, Alg-AmB and Alg-AmB + NCC. 

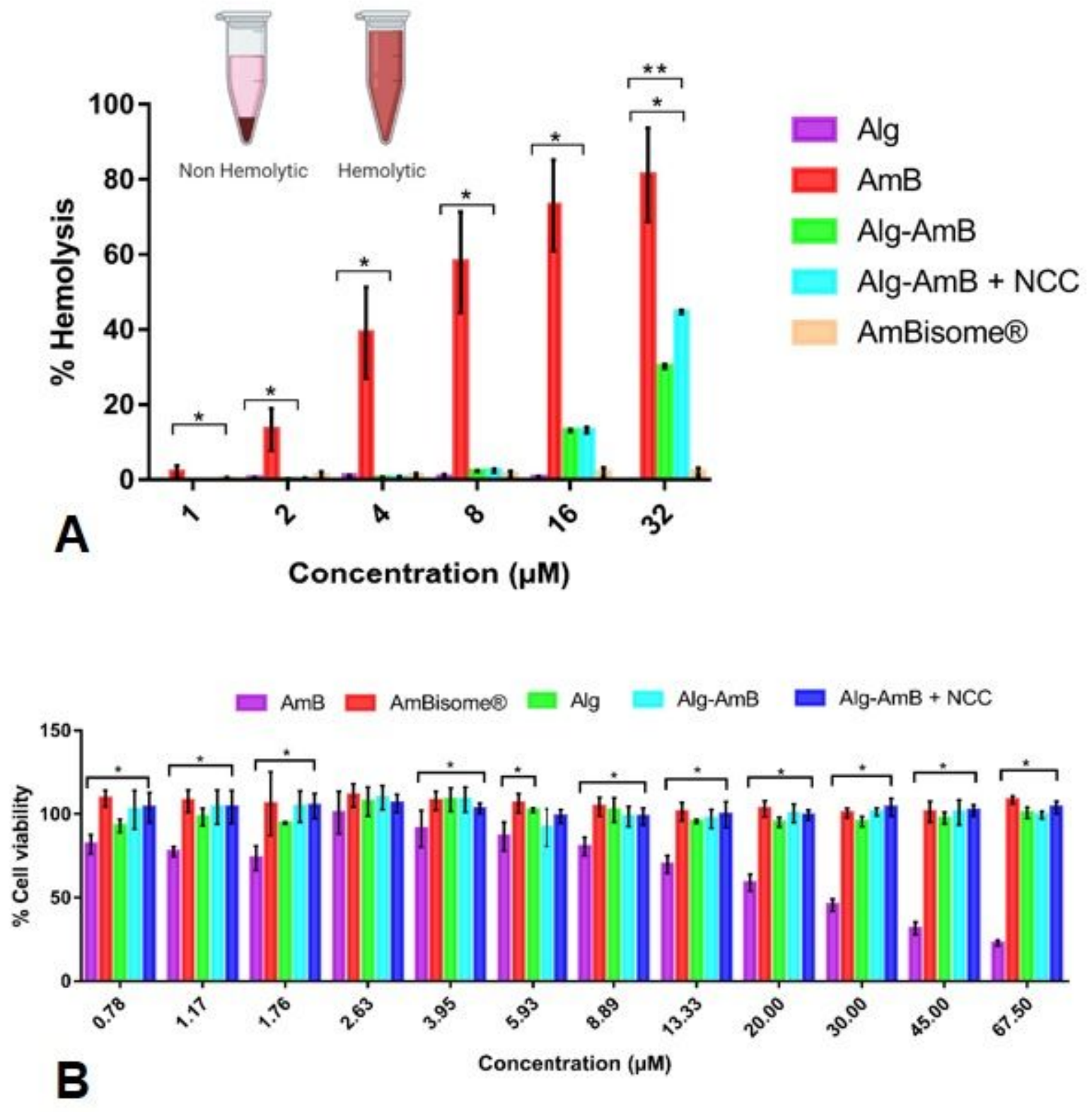

Figure 2

Percentage of hemolysis after treatment of red blood cells (A) Cytotoxicity of the renal cell line HEK293T (B) with Alg, Alg-AmB, Alg-AmB + NCC, AmBisome ${ }^{\circledR}$ and AmB. Note: A $\neg-A m B\left({ }^{\star}\right)$ and Alg-AmB (**) were compared to the other groups by the Duncan test $(p<0,05)$. B - AmB $\left(^{*}\right)$ was compared to the other groups by the Duncan test $(p<0.05)$. 